\title{
The effect of accessory auditory stimulation upon detection of visual signals*
}

\author{
DENNIS M. MALONEY† and ROBERT B. WELCH \\ University of Kansas, Lawrence, Kans. 66044
}

The study examined the effect of a continuous $1,000-\mathrm{Hz} 80-\mathrm{dB}$ accessory tone upon the detectability of a visual stimulus presented to the peripheral retina. All Ss received the primary visual stimulus both with and without an accessory auditory stimulus. Detection of the visual stimulus was measured by means of a forced-choice indicator. The presence of the accessory tone significantly decreased the percentage of light detections. An unexpected finding was that incorrect responses were unequally distributed among the four observation intervals. It was concluded that continuous intense accessory auditory stimulation inhibits visual sensitivity.

Psychologists have long been interested in the problem of how sensory stimuli of different modalities interact with each other when presented simultaneously. Soviet investigators have examined sensory interaction (London, 1954), as have Western researchers (Gilbert, 1941; Ryan, 1940). Recently, interest has focused almost exclusively upon interactions between the auditory and visual modalities (e.g., Loveless, Brebner, \& Hamilton, 1970). One paradigm entails the measurement of percent detections of a visual stimulus (primary modality) both with and without an auditory (accessory) stimulus present (Watkins, 1964; Watkins \& Feehrer, 1964, 1965).

It is not clear whether auditory stimulation facilitates or inhibits visual detections or if the effects are actually the result of distraction. Some investigators (e.g., Thorne, 1934) have reported facilitation of vision for some Ss and inhibition for others. On the other hand, a number of investigations have revealed less equivocal results. Davis (1966) reported that accessory auditory stimulation led to a decrease in visual detections. Conversely, several studies have obtained increased detectability as their sole effect (Watkins, 1964; Watkins \& Feehrer, $1964,1965)$. It is significant that in these studies the conditions with the longer sound durations induced considerably less facilitation. Thus, it is possible that duration of the accessory stimulus is an important determinant of its effect upon vision. However, other parameters appear to be involved as well. For instance,

*The experiment described formed the basis of a thesis leading to the MA degree from the Department of Psychology at the University of Kansas. The authors wish to express their gratitude to Drs. George Kellas and Juliet Shaffer for their assistance.

+ Requests for reprints should be sent to the Department of Psychology, University of Kansas. Lawrence. Kans. 66044 .
London (1954) discussed the results of Soviet investigations which indicated that the tonal frequency of the auditory stimulus must be taken into account. Maruyama (1959) confirmed this finding and showed, in addition, that the intensity of the sound is an important factor.

Davis's (1966) report of sound-induced inhibition of vision has been termed a "highly contradictory finding" (Loveless, Brebner, \& Hamilton, 1970). While the two important parameters of sound frequency. and intensity were not greatly different from those employed by others, the duration of the accessory auditory stimulus was unusually long. Thus, it seems likely that the visual inhibition reported by Davis was due largely to the long accessory stimulus duration. As suggested by Ince's (1968) findings, it is further hypothesized that the chance of inhibition occurring would be greater when using higher sound intensities. The present study used an intense long-lasting accessory auditory stimulus of an intermediate wave frequency. These conditions were considered optimal for producing a sound-induced inhibition of visual detections.

\section{DESIGN}

Two groups were measured on their ability to detect a threshold-level visual stimulus presented to a locus on the peripheral retina. During alternating trial blocks, a high-intensity auditory stimulus was also present. The groups differed with regard to the order in which the sound and no-sound trials occurred. Order effects were controlled within Ss by repeating the reversal of conditions three times per S (Sidman, 1960) and between $S$ s in the usual manner by presenting the two groups with a reversed pattern of sound and no-sound trial blocks (Hays, 1963). SUBJECTS

The Ss were college students (16 males and 16 females) who participated in the experiment to fulfill a course requirement. They were divided into two groups of 16 Ss each. APPARATUS

The $\mathrm{S}$ sat inside a dark $1.83 \times .91 \times$ $1.83 \mathrm{~m}$ enclosed chamber and looked across a table at a vertical panel containing various lights. A chinrest, $77 \mathrm{~cm}$ from the panel, was fastened to the table. The target disk was located on the panel directly in front of $S$ and subtended a visual angle of approximately $4 \mathrm{deg}$. Illumination of the target disk could be varied by means of a polymetric brightness comparator (Model V-0659). Three red lights in a horizontal row, $4 \mathrm{~cm}$ apart, were positioned $16 \mathrm{~cm}$ above the target disk. The central of these lights served as a fixation point and was situated so that the target disk illuminated S's retina at an angle of incidence 12 deg dorsal to the fovea. A black sleeve tied around S's left arm permitted access to a hidden response button located outside the dark chamber. The $\mathrm{S}$ wore Lafayette stereo headphones (Model F-767). A $1,000 \cdot \mathrm{Hz} 80 \cdot \mathrm{dB}$ (re .0002 dynes $/ \mathrm{cm}^{2}$ ) tone was generated by a Beltone audiometer (Model 12-A). Three 60 -sec automatic timing control timers were used in conjunction with a relay panel and four silent switches to control the duration of the stimuli. The apparatus was activated by a Heathkit regulated power supply (Model IP-20).

The average ambient noise level of the chamber was $60 \mathrm{~dB}$ (re .0002 dynes $/ \mathrm{cm}^{2}$ ), as measured by a Bruel \& Kjaer sound pressure level meter (Model 2203). The thick padding of the headphones served to shield $\mathrm{S}$ from extraneous sounds. PROCEDURE

The $\mathbf{S}$ participated in two sessions, each lasting approximately $1 \mathrm{~h} 50 \mathrm{~min}$. The first $10 \mathrm{~min}$ of a session were used to achieve partial dark adaptation.

A session comprised 10 blocks of 10 trials each. A trial consisted of four 5 -sec observation intervals (OIs), each of which was followed by a 5-sec pause. The trial concluded with a $7-\mathrm{sec}$ period, during which $S$ responded. A 53 -sec rest period occurred after each block of 10 trials. The onset of an OI, a response period, or a rest period was signaled to $\mathrm{S}$ by the illumination of the appropriate red light (or lights) above the target disk.

The task was to decide during which of the four OIs the target disk had been illuminated. The $\mathrm{S}$ indicated his choice during the response period by pressing a button once for $\mathrm{OI}_{1}$, twice for $\mathrm{OI}_{2}$, and so forth. The $S$ was required to respond after every trial regardless of his certainty. The particular OI that contained the 


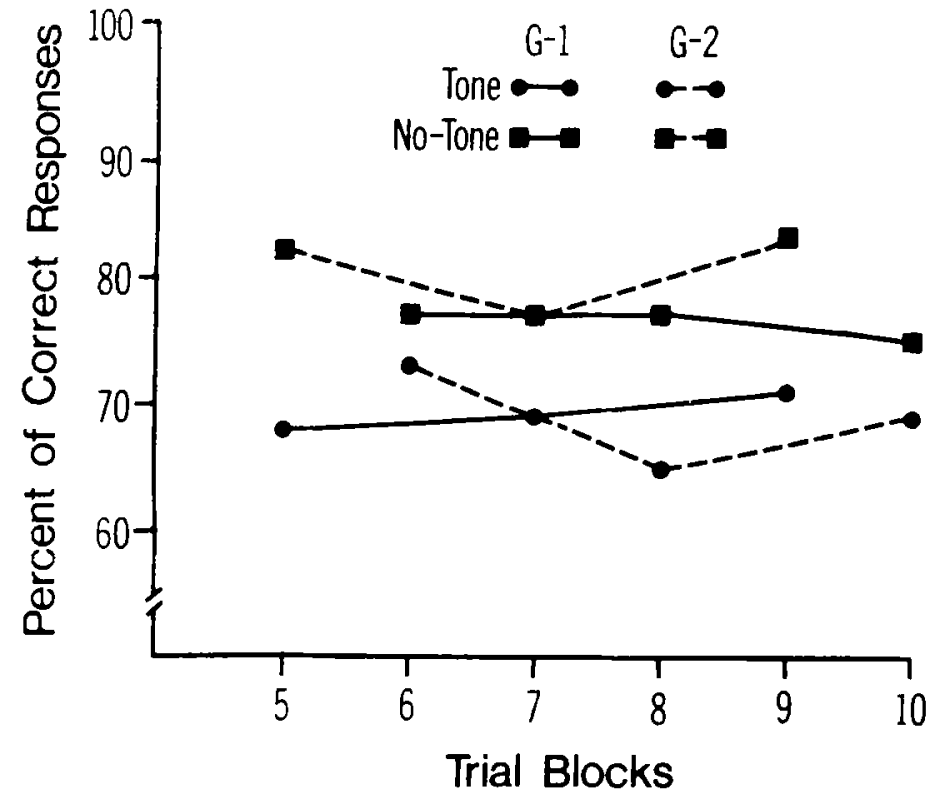

Fig. 1. The mean percentages of correct responses for each of the last six trial blocks of Session 2 for Groups 1 and 2 (G-1 and G-2).

illuminated disk on a given trial was varied in a random manner for each $S$.

During Session $1, \mathrm{~S}$ was given extended practice on the task and an estimate of his visual threshold was made. Visual intensities at 10 different levels were presented randomly without a concomitant auditory stimulus. The intensity that $\mathbf{S}$ was able to detect $60 \%$ to $80 \%$ of the time was defined as his threshold. This level served as a starting point for the selection of visual intensities for Session 2. In this second session, intensities at and near this value were presented during the first two blocks of trials. On the basis of S's performance during these trials, an intensity value was chosen to be presented during Blocks 3 and 4 . Only if $\mathrm{S}$ detected this stimulus $60 \%$ to $80 \%$ of the trials during these two blocks was $h$ is subsequent performance retained for analysis (nine $\mathrm{Ss}$ failed to meet this criterion). This intensity was thus considered to be at threshold value and was used for the remaining six trial blocks. During half of these blocks, the accessory auditory stimulus was also present. The continuous binaural tone occurred in Blocks 5, 7, and 9 for Group 1 and Blocks 6, 8, and 10 for Group 2. This tone began with the initiation of the first trial of the appropriate block and remained on throughout all 10 trials (a period of $7 \mathrm{~min} 50 \mathrm{sec}$ ).

\section{RESULTS}

The main dependent measure was the percentage of correct responses (visual detections) that $\mathrm{S}$ made over the 10 trials in each of the last six trial blocks of Session 2. Thus, $\mathrm{S}$ has six percentage scores-three for the tone blocks and three for the no-tone blocks. The mean percentages of correct responses for all conditions are contained in Fig. 1.

A three-factor mixed analysis of variance (Groups by Tone/No Tone by Trial Blocks) was performed. Only the tone/no-tone factor proved statistically significant $[F(1,30)=$ $18.10, \mathrm{p}<.001]$; the accessory auditory stimulus decreased the detectability of the visual stimulus.

In order to assess the possibility (first pointed out by Symons, 1963) that the tone affected variability in signal detection, an analysis of variance was carried out on variance scores. However, because variability of percentage scores is not independent of their mean, the Freeman-Tukey modification of the arcsine transformation (Lindzey, 1954) was performed on the percentages prior to calculation of the variance scores for the analysis. This formula is:

transformed score

$$
=\frac{\sin ^{-1} \sqrt{\frac{x}{n+1}}+\sin ^{-1} \sqrt{\frac{x+1}{n+1}}}{2}
$$

where $\mathrm{x}=$ percent of correct responses and $n=$ number of trials. A constant of 1 was added to these variances (three of which equaled zero), and the logarithms of the transformed variance scores were then computed. Logarithms were used to normalize the skewed distribution of the difference scores (no-tone variances minus the tone variances). A two-factor analysis of variance (Groups by Tone/No Tone) was then performed on the logarithms of the variances. Neither the group factor $[F(1,30)=.07$, $p>.05]$ nor the tone/no-tone factor $[\mathrm{F}(1,30)=.19, \mathrm{p}>.05]$ proved significant.

The distribution of incorrect responses across the four OIs was examined in order to determine if a response bias existed. The relationship between the percentages of incorrect responses, correct responses, and visual stimulus presentations is presented in Fig. 2. The percentages of both groups are combined, since they produced almost identical curves.

It can be observed from Fig. 2 that the distribution of the percentage of incorrect responses was considerably different from the other two curves. It should also be noted that although the percentages of visual stimulus presentations were not exactly $25 \%$ per OI, the discrepancy was small for each of the four OIs.

\section{DISCUSSION}

The major finding of the present experiment was that a loud continuous tone decreased the detectability of a peripheral visual stimulus. This finding is consistent with the results reported by Davis (1966) and confirmed the present hypothesis, i.e., a long-lasting intense auditory stimulus will inhibit visual detections.

It should be noted that conditions involving accessory auditory stimuli of shorter durations and lower intensities than those of the present study have most often resulted in an increase in visual detectability (Maruyama, 1959; Watkins, 1964; Watkins \& Feehrer, 1964,1965 ). The results of this study, then, when considered together with previous investigations, suggest that duration is indeed an important parameter in sensory interaction. Both tonal frequency and sound intensity have already been shown to be significant variables (London, 1954; Maruyama, 1959).

A physiological basis for the inhibitory effect of an accessory auditory stimulus on vision has been suggested by Davis (1966). He theorized that such an effect could be mediated by certain inhibitory neurons which are activated if the accessory stimulus is very intense. These inhibitory neurons were postulated to be in close contact with neurons of the visual pathway.

It has been suggested (e.g., Broadbent, 1958) that an accessory stimulus may have as at least one of its effects a tendency to distract the $S$ during a detection task. Symons 
(1963) assumed that this distraction would manifest itself as an increase in within-S variability in performance. In neither his nor the present investigation was such a change in variability found. Thus, it can be concluded that the visual inhibition demonstrated in the present study was not merely the result of distraction.

An unanticipated finding was that incorrect responses were unequally distributed among the four OIs. At the end of each trial, the $S$ tended to select either $\mathrm{OI}_{1}$ or $\mathrm{OI}_{2}$ much more frequently than the remaining two OIs. This preference was not related to the percentages of correct responses or visual presentations for the four OIs. It is possible that the $\mathrm{S}$ adopted the "response effort" strategy of rarely pressing the button more than twice when no visual signal was detected. Further research will be necessary to determine the validity of this hypothesis.

In conclusion, the results of the present study indicate that an intense auditory stimulus presented for a prolonged duration will inhibit visual sensitivity. Needed are parametric studies to investigate systematically the variables of intensity, duration, and frequency. of the auditory accessory stimulus.

\section{REFERENCES}

BROADBENT, D. E. Perception and communication. New York: Pergamon Press, 1958.

DAVIS, E. Heteromodal effects upon visual thresholds. Psychological Monographs, 1966,80 (Whole No.633)

GILBERT, G. Intersensory facilitation and

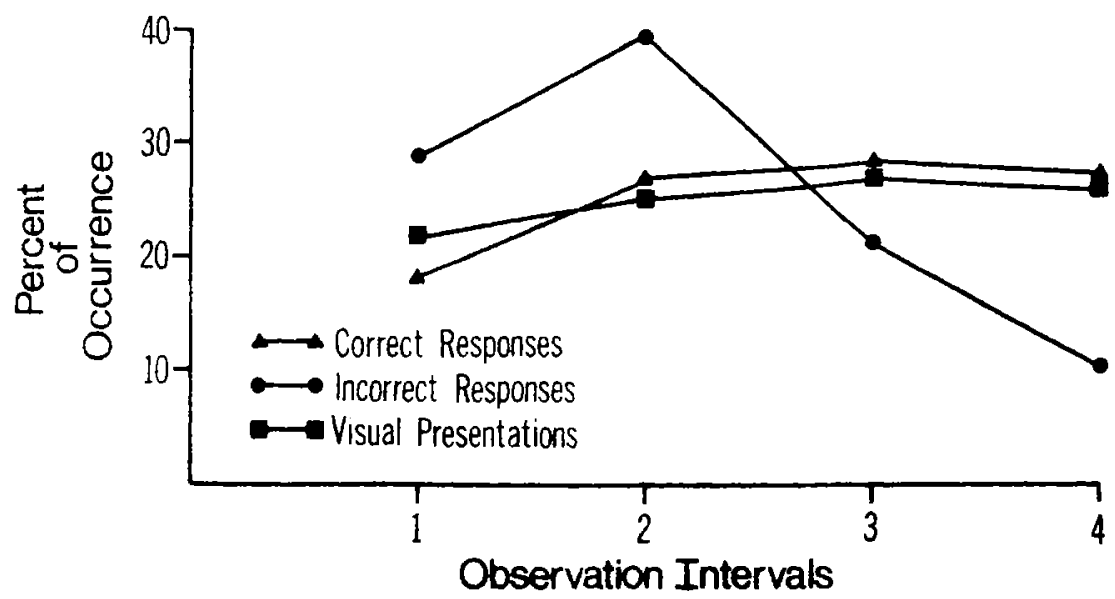

Fig. 2. The mean percentages of incorrect responses, correct responses, and visual stimulus presentations for the four OIs during Session 2 .

inhibition. Journal of General Psychology, ig41,24,381-409.

HAYS, W. L. Statistics. New York: Holt, Rinehart \& Winston, 1963.

INCE,L. Effects of low-intensity acoustical stimulation on visual thresholds. Perceptual \& Motor Skills, 1968, 26, $115-121$.

LINDZEY, G. (Ed.) Handbook of social psychology. Vol. 1. Cambridge, Mass: Addison-Wesley, 1954

LONDON, I. Research in sensory interaction in the Soviet Union. Psychological Bulletin, 1954, 51 , 531-568.

LOVELESS, N., BREBNER, J., \& HAMILTON, P. Bisensory presentation of information. Psychological Bulletin, 1970,73, 161-199.

MARUYAMA, K. Effect of intersensory tone stimulation on absolute light threshold. Tohoku Psychologica Folio, $1959,17,51-81$.

RYAN, $T$. Interrelations of the sensory systems in perception. Psychological
Bulletin, 1941, 37, 659-698.

SIDMAN, M. Tactics of scientific research. New York: Basic Books, 1960.

SYMONS, J. R. The effect of various heteromodal stimuli on visual sensitivity. Quarterly Journal of Experimental Psychology, 1963, 15, 243-251.

THORNE, F. The psychophysical measurement of the temporal course of visual sensitivity. Archives of Psychology, $1934,25,1-66$

WATKINS, W. Effect of certain noises upon detection of visual signals. Journal of Experimental Psychology, 1964, 67. $72-75$.

WATKINS, W., \& FEEHRER, C. Investigations of acoustic effects upon visual signal detection. Technical Report No. 64-577, December 1964, USAF Electronics Systems Division, Bedford, Mass.

WATKINS, W. \& FEFHRER, C. A coustic facilitation of visual detection. Journal of Experimental Psychology, 1965, 70. 332-333. 